Amsterdam, The Netherlands; '2Erasmus Medical Center, Rotterdam, The Netherlands; ${ }^{3}$ Academic Medical Center, Amsterdam, The Netherlands; ${ }^{4} \mathrm{MC}$ Haaglanden Hospital, The Hague, The Netherlands; ${ }^{5}$ The Hague Municipal Health Service, The Hague, The Netherlands; ${ }^{6}$ Municipal Health Service Amsterdam, Amsterdam, The Netherlands, ${ }^{7}$ National Institute for Public Health and the Environment, Bilthoven, The Netherlands, ${ }^{8}$ Anova Health Institute, Tzaneen, South Africa

Background Chlamydia trachomatis is the most prevalent bacterial sexually transmitted infection worldwide. A strong link between serogroup/serovar and serological response has been suggested previously. This study aims to elucidate serovar specific serological responses in two independent Dutch patient groups using two serological assays. Methods We performed genotyping of serovars in two patient groups of C. trachomatis infected patients (total $n=718$ ). We pooled the two study populations to form one study group and within this group we analysed men and women separately. We used two commercially available ELISA kits (medac Diagnostika) to determine specific serum IgG levels.

Results Calculations of the kits to determine IgG concentrations were comparable and could therefore be pooled. We observed very significant differences when comparing the mean $\operatorname{Ig} G$ titres of three serogroups, B, C, and I. In the female group B vs. C: $p<0.0001$ (mean titres B 270.0 vs. C 88.8); B vs. I: $p<0.0001$ (270.0 vs. 108.5). Male group B vs. C: $p=0.0005$ (190.2 vs. 69.6); B vs. I: $p=0.0002$ (190.2 vs. 92.9); C vs. I was not significant. Serovars D and E of serogroup $B$ induce the highest mean IgG titres compared to the other serovars in both men and women: 145.5 and 199.1 vs. $\leq 107.9$ for men and 305.6 and 262.7 vs. $\leq 161.5$ for women.

Conclusions This study shows a statistically significant higher serological response induced by $\mathrm{B}$ group serovars compared to the $\mathrm{C}$ and I group serovars in vivo in both men and women. This study is currently being extended with a different ethnical population and a different serological test.

\section{P1.005 EVALUATION OF CYTOKINES AND MATRIX METALLOPROTEINASES GENES EXPRESSION IN GENITAL ORGANS AFTER VAGINAL EXPOSURE TO CHLAMYDIA MURIDARUM}

doi:10.1136/sextrans-2013-051184.0226

\begin{abstract}
A Marangoni, ${ }^{2} \mathrm{C}$ Cavallini, ${ }^{1} \mathrm{C}$ Foschi, ${ }^{1} \mathrm{P}$ Nardini, ${ }^{3} \mathrm{R}$ Aldini, ${ }^{4} \mathrm{~A}$ D'Errico, ${ }^{4} \mathrm{~F}$ Rosini, ${ }^{1} \mathrm{R}$ Cevenini. 'University of Bologna, Microbiology, DIMES, Bologna, Italy; ${ }^{2}$ University of Bologna, Laboratory of Molecular Biology and Stem Cell Engineering, DIMES, Bologna, Italy; 'University of Bologna, FaBit Dpt., Bologna, Italy; ${ }^{4}$ University of Bologna, DIMES, Bologna, Italy
\end{abstract}

Background Although the pathologic consequences of Chlamydia genital infection are well-established, the mechanisms leading to tissue damage are not completely understood.

Methods All the experiments were approved by the Ethical Committee of the University of Bologna. Animals used were 24 female $\mathrm{Balb} / \mathrm{c}$ mice, 7 weeks old. All animals received medroxyprogesterone acetate 9 and 2 days prior the infection.

Twelve mice were infected by placing $15 \mu$ l of sucrose-phosphateglutamic acid (SPG) buffer containing $10^{6}$ inclusion forming units (IFUs) of C. muridarum into the vaginal vault. Nine animals were inoculated with $15 \mu \mathrm{l}$ of SPG containing heat-inactivated $10^{6}$ IFUs of $C$. muridarum. As controls of inflammation, 3 animals were challenged with $15 \mu$ l of SPG.

At 3,10 , and 20 days post-infection 4 infected animals, 3 animals inoculated with heat-inactivated bacteria and 1 control were sacrificed.

Genital tracts were divided into the cervical-vaginal region, uterine horns, and oviducts.

Right uterine horns and oviducts were stored in formalin and later processed for histological examinations. The remaining parts of the organs were used for RNA extraction, by using Trizol Reagent (Invitrogen), in combination with RNeasy Mini Kit (Qiagen).
cDNA was synthesised with SuperScript III RT (Invitrogen). Real-time RT-PCR was performed with SYBR Green Fast Start kit (Roche Diagnostics). Primers used to assess INF- $\gamma$, TNF- $\alpha$, MMP-2, MMP-9 and GAPDH levels were from SuperArray (SABiosciences).

Results At histological examination no controls showed inflammation. On the contrary, scores of inflammation in all the organs from infected animals peaked at day 10 , whereas only a single animal inoculated with inactivated bacteria showed a very mild inflammation at day 10 in its uterus.

At day 10, organs from infected animals showed significantly higher MMP-2 and MMP-9 genes expression than organs obtained from non-infected mice.

Conclusions Our study confirms the pivotal role of MMPs in the development of tissue damage.

\section{P1.006 THE EFFECTS OF SYPHILIS ON CD4 CELL COUNTS AND PLASMA HIV-1 VIRAL LOADS AMONG PATIENTS WITH HIV- SYPHILIS CO-INFECTION}

doi:10.1136/sextrans-2013-051184.0227

I Levy, Y Maor, V Litachevsky, G Rahav. Sheba Medical Center, Ramat Gan, Israel

Background Concomitant syphilis and human immunodeficiency virus (HIV) infection is increasingly frequent in industrialised countries. We examined the effect of active syphilis on CD4 counts and plasma HIV-1 viral loads

Methods All patients with syphilis-HIV coinfection treated at the Sheba medical centre between 2007 and 2012 were included in the study. Patients were divided to those with early or secondary syphilis and to those with latent syphilis (early and late). CD4 cell counts and plasma HIV-1 viral loads were measured before, during and following syphilis treatment.

Results 17 patients were included: all were men having sex with men, 11 (65\%) were treated with ART: 5 with primary syphilis, 4 patients with secondary syphilis, 2 patient with early latent and 6 patients with latent syphilis of unknown duration ( $r p r>1: 32$ ), one of them with neurosyphilis. Median CD4 cell count significantly dropped during syphilis by $25 \%$ : from 575 (246-828) before syphilis diagnosis to 415 (195-630) cells $/ \mathrm{mm}^{3}$ during syphilis $(p=0.0002)$. After penicillin treatment it rose back to $621(250$ 1379) $(p=0.01)$.

Plasma VL in 6 of the patients that did not receive ART increased during syphilis, although this rise was not statistically significant.

Conclusions Syphilis was associated with a transient decrease in the CD4 cell count and with an increase in VL in HIV-infected men; This increase in VL, although statistically non sugnificant, may explain at least partially the increased risk of HIV transmission in HIV patients not treated by ART that are co infected with syphilis

\section{P1.007 IMMUNE-RESPONSE IN LESIONAL SKIN OF SECONDARY SYPHILIS}

doi:10.1136/sextrans-2013-051184.0228

M Cusini, D Fanoni, S Ramoni, E Berti. Dermatology Unit - Fondazione IRCCS Ca Granda Ospedale Maggiore Policlinico, Milan, Italy

Background It is well known that tissue immune response plays an important role in the pathogenetic mechanisms of several bacterial and viral infections. Several studies focused on the cutaneous host immune-response during infections by mycobacteria, HIV, HHV8, HCV and HSV, while few data are known about cell-mediated immunity in skin syphilitic lesions.

Methods By an immunohistochemistry technique, we characterised the cutaneous inflammatory infiltrate in 5 cases of secondary syphilis, using a large panel of monoclonal antibodies (MAbs) and a specific polyclonal antibody against Treponema pallidum (TP) antigen. 